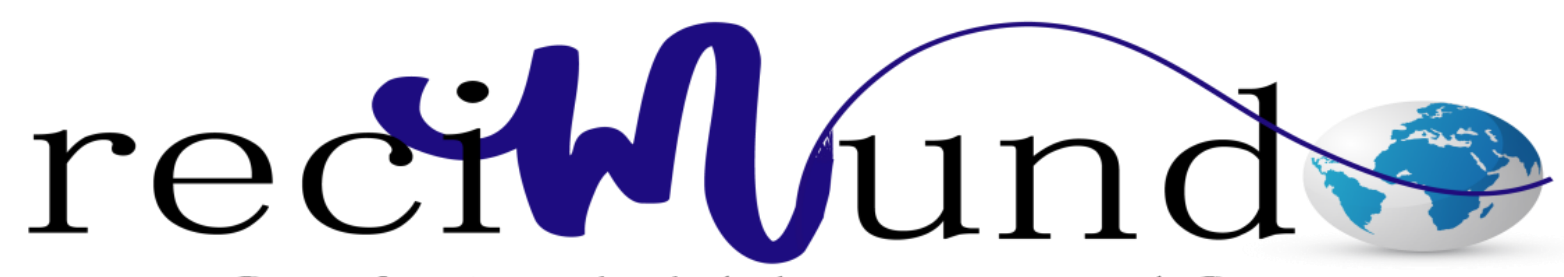

Revista Cientifica Mundo de la Investigación y el Conocimiento

Jenny Jazmín Ortega Flores a ; Andrea Carolina Villao Recalde ${ }^{\text {b }}$; Ximena Angélica

Vargas Arizaga ${ }^{c}$; Johanna Denys Suarez Orrala ${ }^{d}$

Esteatosis y Cirrosis Hepática: Métodos diagnósticos mediante Imagenología

Steatosis and Hepatic Cirrhosis: diagnostic methods through imagenology

Revista Científica Mundo de la Investigación y el Conocimiento. Vol. 3 núm.1, enero, ISSN: 2588-073X, 2019, pp. 75-89

DOI: 10.26820/recimundo/3.(1).enero.2019.75-89

URL: http://www.recimundo.com/index.php/es/article/view/358

Editorial Saberes del Conocimiento

Recibido: $20 / 11 / 2018$

Aceptado: 05/01/2019

Publicado: 31/01/2019

Correspondencia: director@ recimundo.com

a. Médico de la Universidad de Guayaquil.

b. Médico de la Universidad de Guayaquil.

c. Médico de la Universidad de Guayaquil.

d. Médico de la Universidad de Guayaquil. 


\section{Esteatosis y Cirrosis Hepática: Métodos diagnósticos mediante Imagenología Vol. 3, núm. 1., (2019) \\ Jenny Jazmín Ortega Flores; Andrea Carolina Villao Recalde; Ximena Angélica Vargas Arizaga; Johanna Denys Suarez Orrala}

\section{RESUMEN}

El Hígado Graso No Alcohólico (HGNA) es una de las hepatopatías más comunes en países industrializados; la presencia de diabetes tipo 2, obesidad, hiperlipidemia y enfermedad cardiovascular son los factores de riesgo de mayor importancia y los más citados durante el establecimiento y desarrollo de la enfermedad de hígado graso, técnicamente llamado esteatosis hepática. Esta enfermedad puede preceder al diagnóstico de diabetes, hipertensión arterial o dislipidemia; su evaluación resulta fundamental para identificar pacientes de riesgo, no solo de fibrosis y cirrosis hepática, sino también pacientes con mayor riesgo de eventos cardiovasculares. Usualmente asintomática, puede producir dolor en el hipocondrio derecho u otros trastornos, que son ignorados por los pacientes. El método diagnóstico de mayor utilidad, es la ecografía considerado el mejor examen imagenológico para detectar la esteatosis hepática en forma precoz ya que ofrece múltiples ventajas. Entre los resultados se obtuvo que el hígado graso es muy común y que el mismo puede ser causado por una variedad de condiciones no necesariamente asociadas a la ingesta crónica de alcohol. Se concluyó que la elastografía por resonancia magnética es un método no invasivo y eficaz, cuyos resultados tienen concordancia con la biopsia hepática, presentando superioridad en comparación con métodos como el de elastografía por ultrasonido, debido a que evalúa un volumen mucho mayor de tejido hepático y muestra la distribución de las lesiones que, a menudo, es heterogénea.

Palabras claves: Enfermedad; Hepática; Grasa; Diabetes; Ecografía; Esteatosis e Imágenes. 


\title{
Esteatosis y Cirrosis Hepática: Métodos diagnósticos mediante Imagenología
}

Vol. 3, núm. 1., (2019)

Jenny Jazmín Ortega Flores; Andrea Carolina Villao Recalde; Ximena Angélica Vargas Arizaga;

Johanna Denys Suarez Orrala

\begin{abstract}
The Non-Alcoholic Fatty Liver (NAFLD) is one of the most common liver diseases in industrialized countries; the presence of type 2 diabetes, obesity, hyperlipidemia and cardiovascular disease are the most important risk factors and the most cited during the establishment and development of fatty liver disease, technically called hepatic steatosis. This disease can precede the diagnosis of diabetes, high blood pressure or dyslipidemia; its evaluation is essential to identify patients at risk, not only fibrosis and liver cirrhosis, but also patients at higher risk of cardiovascular events. Usually asymptomatic, it can produce pain in the right hypochondrium or other disorders, which are ignored by patients. The most useful diagnostic method is ultrasound, considered the best imaging test to detect hepatic steatosis at an early stage since it offers multiple advantages. Among the results it was found that fatty liver is very common and that it can be caused by a variety of conditions not necessarily associated with chronic alcohol intake. It was concluded that magnetic resonance elastography is a non invasive and effective method, whose results are consistent with liver biopsy, presenting superiority compared to methods such as ultrasound elastography, because it evaluates a much larger volume of liver tissue and shows the distribution of lesions that is often heterogeneous.
\end{abstract}

Key words: Disease; Liver; Fat; Diabetes; Ultrasound; Steatosis and Images. 


\section{Esteatosis y Cirrosis Hepática: Métodos diagnósticos mediante Imagenología Vol. 3, núm. 1., (2019) \\ Jenny Jazmín Ortega Flores; Andrea Carolina Villao Recalde; Ximena Angélica Vargas Arizaga; Johanna Denys Suarez Orrala}

\section{Introducción.}

El hígado graso no alcohólico (HGNA) es una de las hepatopatías más comunes en países industrializados; la presencia de diabetes tipo 2, obesidad, hiperlipidemia y enfermedad cardiovascular son los factores de riesgo de mayor importancia y los más citados durante el establecimiento y desarrollo de la enfermedad de hígado graso.

La acumulación de grasa en el hígado, técnicamente llamado esteatosis hepática, es un problema bastante común que puede ser causado por factores de riesgo como obesidad, diabetes, colesterol alto y consumo excesivo de bebidas alcohólicas.

La esteatosis hepática puede preceder al diagnóstico de diabetes, hipertensión arterial o dislipidemia; su evaluación resulta fundamental para identificar pacientes de riesgo, no solo de fibrosis y cirrosis hepática, sino también pacientes con mayor riesgo de eventos cardiovasculares. (Graffigna, y otros, 2017)

La esteatosis hepática a nivel mundial, presenta tendencias ascendentes, debido a múltiples factores, de allí que las autoridades deben tomar decisiones pertinentes frente al problema, se ha incrementado en los últimos años a niveles alarmantes, sin distinguir a las personas por su edad, sexo o condición social, mientras la mayoría de la población no hace una verdadera conciencia de lo grave que se puede volver este problema si no se corrige con prontitud. La detección precoz de la esteatosis hepática en sus primeras etapas, es de capital importancia ya que puede convertirse en una enfermedad crónica muy grave, producir una cirrosis hepática e inclusive producir la muerte. (Vallejo, Peñafiel, \& Acuña, 2017)

La esteatosis hepática no alcohólica es la más frecuente y afecta al $30-40 \%$ de los hombres y al $15-20 \%$ de las mujeres en la población general. Suele estar relacionada con 


\section{Esteatosis y Cirrosis Hepática: Métodos diagnósticos mediante Imagenología}

Vol. 3, núm. 1., (2019)

Jenny Jazmín Ortega Flores; Andrea Carolina Villao Recalde; Ximena Angélica Vargas Arizaga;

Johanna Denys Suarez Orrala

obesidad, resistencia a la insulina o síndrome metabólico generando acumulación de lípidos y una respuesta inflamatoria que puede derivar en cirrosis. (Padrón, 2017)

Usualmente asintomática, puede producir dolor en el hipocondrio derecho u otros trastornos, que son ignorados por los pacientes. El método diagnóstico de mayor utilidad, es la Ecografía considerado el mejor examen imagenológico para detectar la esteatosis hepática en forma precoz ya que ofrece múltiples ventajas, y permite realizar rastreos de alta sensibilidad y especificidad al detectar la esteatosis hepática, evidenciando la infiltración grasa del hígado, cuando produce incremento difuso de su ecogenicidad, comparada con la de los riñones. (Vallejo, Peñafiel, \& Acuña, 2017)

Se considera fundamental hacer mención a lo expuesto por Zanin, puesto que ésta especialista clasifica de manera clara los grados de acumulación de grasa en el hígado, lo que entonces, en el caso que nos ocupa, sirve como contexto general donde ubicar de manera práctica a la esteatosis hepática, presente en dos etapas (grados) de acumulación de grasa hepática, que son:

- Grado 1 o esteatosis hepática simple: es un exceso de grasa en el hígado considerado inofensivo. Generalmente el paciente no presenta síntomas y solo descubre el problema a través de un examen de sangre de rutina.

- Grado 2 o esteatosis hepática no alcohólica: es aquella en la que, además del exceso de grasa, el hígado se inflama. Generalmente el paciente presenta síntomas como dolor en el lado derecho del abdomen y la barriga hinchada.

- Grado 3 o fibrosis hepática: en este caso existe grasa e inflamación que causa alteraciones en el órgano y en los vasos sanguíneos a su alrededor, pero el hígado todavía funciona 


\section{Esteatosis y Cirrosis Hepática: Métodos diagnósticos mediante Imagenología}

Vol. 3, núm. 1., (2019)

Jenny Jazmín Ortega Flores; Andrea Carolina Villao Recalde; Ximena Angélica Vargas Arizaga; Johanna Denys Suarez Orrala

normalmente.

- Grado 4 o cirrosis hepática: es la fase más grave de la enfermedad y surge después de años de inflamación, siendo caracterizada por la alteración en todo el hígado que causa reducción de su tamaño y dejan su forma irregular. La cirrosis puede evolucionar para cáncer o muerte del hígado siendo necesario en estos casos trasplantar el órgano.

La autora destaca que “...además de evaluar la cantidad de grasa en el hígado, también es importante verificar la presencia de inflamación, ya que es la principal causa de muerte de las células de este órgano". Refiere que, para evaluar la progresión de la enfermedad, se puede utilizar la elastografía hepática, que es un examen rápido y sin dolor. (Zanin, 2018)

El diagnóstico es realizado principalmente por métodos imagenológicos no invasivos. La ecografía abdominal es el estudio de imágenes utilizado con más frecuencia para identificar hígado graso debido a su gran disponibilidad, seguridad, no invasivo, bajo costo y exactitud en el diagnóstico. El procedimiento diagnóstico compara la ecogenicidad del riñón derecho con el parénquima hepático. El hígado sin esteatosis muestra una ecoestuctura homogénea similar a la corteza renal y al parénquima esplénico. El hígado graso tiene una ecogenicidad mayor (más brillante) que la corteza renal y el bazo, éste último debido a la acumulación intracelular de vacuolas grasas. La ecografía es útil para diagnosticar esteatosis de grados 2 y 3 pero es menos efectiva para grado 1. Por otra parte, no permite distinguir entre fibrosis y esteatosis hepática. (ANMM, 2015)

La tomografía computarizada tiene una sensibilidad de $82 \%$ y una especificidad de $100 \%$ para diagnosticar esteatosis hepática cuando el contenido de grasa es $\geq 30 \%$. Aunque la TC es un método rápido, no operador dependiente, se debe tener en cuenta la radiación a la que se expone 


\section{Esteatosis y Cirrosis Hepática: Métodos diagnósticos mediante Imagenología}

Vol. 3, núm. 1., (2019)

Jenny Jazmín Ortega Flores; Andrea Carolina Villao Recalde; Ximena Angélica Vargas Arizaga;

Johanna Denys Suarez Orrala

a los pacientes. Al igual que la ecografía, la $\mathrm{TC}$ es bastante exacta para diagnosticar esteatosis grado 2-3, pero no para detectar esteatosis grado 1 . Por otro lado, la atenuación del parénquima hepático puede verse afectada por otros factores, como la presencia de exceso de hierro y glucógeno, drogas como la amiodarona y el metotrexate o hepatitis tóxica aguda y cirrosis. (ANMM, 2015)

La resonancia magnética es el método de diagnóstico por imágenes más sensible para detección del incremento de grasa intrahepática. Se ha encontrado buena correlación entre RM e histología en pacientes con esteatosis hepática y puede detectar bajos contenidos de grasa intrahepática.

En la presente investigación documental se pretende definir, describir y explicar los aspectos básicos relacionados a esta patología, teniendo presente que el objetivo es construir un valioso material de apoyo para otras investigaciones más profundas y a su vez sirva de contenido didáctico sustancioso en general para los interesados en el tema. Entre los puntos a tratar tenemos: clasificación de los grados de acumulación de grasa en el hígado, concepto de esteatosis hepática, causas, factores de riesgo, síntomas, diagnóstico imagenológico, tratamiento y relación existente con la cirrosis hepática.

\section{Materiales y Métodos.}

En el presente estudio se han desarrollado puntos básicos relacionados con la esteatosis hepática, centrando estos fundamentalmente en la definición de la mencionada condición, causas, síntomas, diagnóstico imagenológico, tratamiento y la relación que esta patología tiene con la cirrosis hepática. 


\section{Esteatosis y Cirrosis Hepática: Métodos diagnósticos mediante Imagenología}

Vol. 3, núm. 1., (2019)

Jenny Jazmín Ortega Flores; Andrea Carolina Villao Recalde; Ximena Angélica Vargas Arizaga; Johanna Denys Suarez Orrala

Para su desarrollo se realizó una investigación de tipo documental. Este método se define como la recolección de documentos, categorizarlos, analizarlos; para después poder presentar un resultado coherente. El objetivo principal de esta metodología es el aporte de nuevos conocimientos sobre el objeto de estudio. (Sampieri, 1998)

Este método se puede resumir en las siguientes características: la recolección, selección, análisis y presentación de información coherente a partir del uso de documentos; la realización de una recopilación apropiada de datos e información que permiten redescubrir hechos, sugerir problemas, orientar hacia otras fuentes de investigación, orientar formas para elaborar instrumentos de investigación, elaborar hipótesis, entre otros factores. Se puede agregar que se debe realizar de forma sistemática y con objetivos precisos, con la finalidad de ser base para la construcción de conocimientos; en él se usan de diferentes técnicas e instrumentos para la localización y categorización de datos, para culminar con el análisis de documentos y de contenidos. (Martínez, 2002)

\section{Resultados.}

Décadas atrás se creía que la acumulación de grasa en el hígado era causada solamente por el consumo exagerado de bebidas alcohólicas, y que la presencia de la esteatosis hepática era necesariamente algo perjudicial a la salud. Idea distinta es la que se defiende en la actualidad, ya que el hígado graso es muy común y que el mismo puede ser causado por una variedad de condiciones no necesariamente asociadas a la ingesta crónica de alcohol. Así mismo, el autor deja claro que "cuanto más grande y más prolongada es la acumulación de grasa en el hígado, mayores son los riesgos de lesión hepática" y que "...la esteatosis hepática es nivel anterior al 


\section{Esteatosis y Cirrosis Hepática: Métodos diagnósticos mediante Imagenología}

Vol. 3, núm. 1., (2019)

Jenny Jazmín Ortega Flores; Andrea Carolina Villao Recalde; Ximena Angélica Vargas Arizaga;

Johanna Denys Suarez Orrala

desarrollo de la esteato-hepatitis, que como el propio nombre dice, nada más es que una hepatitis causada por exceso de grasa". (Pinheiro)

Actualmente se conoce que la causa principal de formación del hígado graso es la captación de los ácidos grasos libres y su depósito en el hígado. Si esta situación evoluciona y se produce un fenómeno inflamatorio hepático, se produce la esteatohepatitis, pudiendo progresar a cirrosis si la inflamación crónica se transforma en fibrosis. El mecanismo de filtro del hígado se vería alterado en fases muy avanzadas. (Mayo Clinic)

Teniendo como base lo afirmado en Mayo Clinic, se puede decir que, tanto el hígado graso no alcohólico como la esteatosis hepática no alcohólica están relacionados con los siguientes factores: Sobrepeso u obesidad, resistencia a la insulina, un nivel elevado de glucemia (hiperglicemia) que indica prediabetes o diabetes tipo 2, niveles altos de grasas en la sangre, en particular los triglicéridos. (Mayo Clinic)

Asimismo, Padrón realiza un aporte en cuanto a los patrones radiológicos de esteatosis hepática, y al respecto explica que entre los tipos que se pueden encontrar están:

- Esteatosis hepática focal: generalmente se debe a variaciones en el aporte vascular conocidos como tercer aporte. Podemos verlo como un área geográfica típicamente en la fosa vesicular, región subcapsular, adyacente a la vena porta o al ligamento falciforme. También puede verse como una pseudomasa sin efecto de masa sobre las estructuras vasculares y puede no ser detectable con TC, en cuyo caso el diagnóstico se realiza con RM.

- Esteatosis hepáticadifusa: es la forma más común y puede verse en todas las causas de EH. 


\section{Esteatosis y Cirrosis Hepática: Métodos diagnósticos mediante Imagenología}

Vol. 3, núm. 1., (2019)

Jenny Jazmín Ortega Flores; Andrea Carolina Villao Recalde; Ximena Angélica Vargas Arizaga; Johanna Denys Suarez Orrala

- Esteatosis hepática perilesional, periportalyperivascular: Se ha visto que el consumo de alcohol, la cirrosis alcohólica y la ingesta de corticoides puede inducir esteatosis periportal-perivascular que debe distinguirse de otras patologías como la fibrosis, el edema o la hemorragia.

- Esteatosis hepática subcapsular: puede ser idiopática o secundaria a la administración de insulina en la diálisis peritoneal, debido a la alta concentración de insulina.

- Esteatosis hepática multinodular: vemos múltiples lesiones nodulares distribuidas por el parénquima hepático. El diagnóstico diferencial debe hacerse con linfoma, sarcoidosis, abscesos, candidiasis, hamartomas biliares o las metástasis sobre todo en pacientes con neoplasia conocida. En dicho caso debe realizarse RM para su diagnóstico antes de realizar una biopsia.

- Esteatosis hepática lobar: es una entidad poco frecuente y se debe a la oclusión de la rama portal derecha o izquierda. El diagnóstico con US o TC puede ser complicado siendo la RM diagnóstica con secuencias en fase y fuera de fase. (Padrón, 2017)

En el boletín de la ANMM se deja claro que los estudios por imágenes son métodos no invasivos que permiten la evaluación cualitativa y cuantitativa de la grasa hepática, y hasta el momento se cuenta con la ecografía, la tomografía computada (TC), la resonancia magnética (RM) y la elastografía, sin embargo, el enfoque perseguido en el presente trabajo investigativo es exponer sobre lo que a criterio propio se considera actualizado. (ANMM, 2015)

De igual manera, se menciona a estudios como los de Stoopen-Rometti et al. (2017), ya que, en el mismo, en cuanto a la esteatosis, es destacada la existencia de una técnica, que por su continuo perfeccionamiento se ha vuelto más atractiva, siendo esta la de la elastografía por 


\section{Esteatosis y Cirrosis Hepática: Métodos diagnósticos mediante Imagenología}

Vol. 3, núm. 1., (2019)

Jenny Jazmín Ortega Flores; Andrea Carolina Villao Recalde; Ximena Angélica Vargas Arizaga;

Johanna Denys Suarez Orrala

resonancia magnética (ERM). En dicho estudio se explica que lo trascendente de ésta técnica estriba en que "puede combinarse en forma adicional y durante la misma sesión con otras secuencias de RM (resonancia magnética) que son útiles para cuantificar los infiltrados del parénquima por grasa o hierro, lo cual permite establecer un protocolo de exploración multiparamétrico. (Stoopen-Rometti, y otros, 2017)

Volviendo a lo hallado en el boletín de la ANMM (2017), se tiene la evaluación de la esteatosis hepática por tomografía computada (TC); en este método se depende de los valores atenuados denominados unidades Hounsfield (Hus) del parénquima hepático, sin embargo, en dicho boletín se deja claro que el mejor método tomográfico para calcular la grasa hepática es la TC sin contraste, que permite una evaluación más cuantitativa de la atenuación hepática. El grado de disminución de la atenuación es el mejor determinante del grado de contenido de grasa hepática. (ANMM, 2015)

La resonancia magnética es el método de diagnóstico por imágenes más sensible para detección del incremento de grasa intrahepática. Se ha encontrado buena correlación entre RM e histología en pacientes con EHGNA y puede detectar esteatosis con solo 3\% de contenido graso. El grado de infiltración grasa puede ser calculado con técnicas de cambio químico (CSI) o espectroscopia (MRS). La RM con CSI y con MRS tiene mayor precisión diagnóstica que el ultrasonido y la TC, alcanzando una exactitud cercana al 100 por ciento. Como desventajas se encuentran el elevado costo y la baja disponibilidad de espectroscopía ya que solo los equipos más modernos tienen este hardware, que se utiliza principalmente en estudios de investigación.

Aparte de esto, cabe destacar a Marcano, quien explica que a pesar de que la esteatosis se pueda cuantificar mediante modalidades sensitivas tales como la tomografía computada (TC) y la 


\section{Esteatosis y Cirrosis Hepática: Métodos diagnósticos mediante Imagenología}

Vol. 3, núm. 1., (2019)

Jenny Jazmín Ortega Flores; Andrea Carolina Villao Recalde; Ximena Angélica Vargas Arizaga; Johanna Denys Suarez Orrala

imagen por resonancia magnética (IRM), especialmente ésta por su novedosa técnica de espectroscopia, asegura que con ninguna de ellas tiene la suficiente sensibilidad y especificidad para estudiar dicha enfermedad, y al igual que la ultrasonografía, no pueden distinguir entre la simple esteatosis y la EHNA con o sin fibrosis. Finalmente el especialista afirma que la biopsia hepática es el único examen que permite determinar la diferenciación entre esteatosis simple (acumulación de grasa) y esteatohepatitis (grasa asociada a inflamación y fibrosis). (Marcano, 2015)

Por otra parte, la elastografía funciona de manera similar a un aparato de ecografía, dispone de una sonda emisora-receptora que emite dos tipos de ondas: una onda pulsátil vibratoria que penetra en el tejido hepático, y una onda de ultrasonidos que capta la velocidad a la que se propaga la primera onda. Si la onda vibratoria viaja lentamente quiere decir que el tejido del hígado es elástico, si la onda viaja rápido quiere decir que el hígado es poco elástico o existe fibrosis por lo que el valor final es alto. (Bartres, 2013)

La finalidad de la elastografía del hígado es obtener, en vivo y en forma no invasiva, información acerca de las propiedades mecánicas del parénquima después de someterlo a un estrés que lo deforme. La elastografía puede realizarse por medio de ultrasonidos (EUS) o por medio de resonancia magnética (ERM). (Stoopen-Rometti, y otros, 2017)

La elastografia por resonancia magnética (ERM) obtiene información de los vectores de las ondas que se propagan en 2 o en 3 dimensiones en el parénquima hepático El volumen de tejido analizado en la ERM es, por ello, mucho más representativo de la infiltración del parénquima y reduce errores debidos a la heterogeneidad que pueda tener la fibrosis El examen con ERM no está limitado por la presencia de ascitis o espacios intercostales cerrados. (Bartres, 


\section{Esteatosis y Cirrosis Hepática: Métodos diagnósticos mediante Imagenología}

Vol. 3, núm. 1., (2019)

Jenny Jazmín Ortega Flores; Andrea Carolina Villao Recalde; Ximena Angélica Vargas Arizaga;

Johanna Denys Suarez Orrala 2013)

Con respecto al tratamiento fundamentalmente consiste en bajar de peso y aumentar la actividad física. Otras recomendaciones incluyen evitar el consumo de alcohol y el consumo de medicamentos innecesarios. (Marcano, 2015). En aquellos pacientes con EHGNA sin obesidad, se debe hacer mayor énfasis en el cambio de los componentes de la dieta y no en la disminución de la ingesta calórica, además de favorecer la actividad física. (ANMM, 2015)

\section{Conclusiones y Recomendaciones}

Aunque el hígado graso es por sí mismo una condición benigna de curso asintomático con mínimas probabilidades de progresar, si no se diagnostica, esta condición puede evolucionar de manera silenciosa. (Barba, 2008)

La esteatosis puede presentarse aislada (esteatosis pura) o puede estar asociada a una hepatitis: la esteatosis hepática no alcohólica (EHNA). La esteatosis y la EHNA constituyen las denominadas “esteatohepatitis no alcohólicas” (My Liver Exam. , 2015)

En algunos casos, la esteatosis puede derivar en una fibrosis, llegando a pasar incluso a la fase de cirrosis, de allí entonces la estrecha relación entre los dos estadios extremos de esta enfermedad, es decir, el inicio, considerado como esteatosis hepática y el fin la cirrosis hepática.

Se hace oportuno hacer referencia a los resultados obtenidos en estudios como los de Stoopen-Rometti et al., puesto que de manera concluyente, a través del mismo llegan a determinar, entre otras cosas, que la elastografía por resonancia magnética llega a ser entonces un método no invasivo y eficaz, cuyos resultados tienen concordancia con la biopsia hepática, presentando superioridad en comparación con métodos como el de elastografía por ultrasonido, 


\section{Esteatosis y Cirrosis Hepática: Métodos diagnósticos mediante Imagenología}

Vol. 3, núm. 1., (2019)

Jenny Jazmín Ortega Flores; Andrea Carolina Villao Recalde; Ximena Angélica Vargas Arizaga; Johanna Denys Suarez Orrala

debido a que evalúa un volumen mucho mayor de tejido hepático y muestra la distribución de las lesiones que, a menudo, es heterogénea.

\section{Bibliografía.}

ANMM. (2015). Enfermedad por hígado graso no alcohólico. Obtenido de Boletín de Información Clínica y Terapéutica de la ANMM.: http://www.medigraphic.com/pdfs/facmed/un-2017/un172h.pdf

Barba, J. (2008). Esteatosis hepática, esteatohepatitis y marcadores de lesión hepática. Revista Latinoamericana de Patología Clínica y Medicina de Laboratorio, 55 (4), 216 - 232.

Bartres, C. \&. (2013). Elastografía hepática (Fibroscan®) en hepatología. . Revista Española de Enfermedades Digestivas, 235-240.

Graffigna, M., Catoira, N., Soutelo, J., Azpelicueta, A., Berg, G., Perel, C., . . . Farias, C. M. (2017). Diagnóstico de esteatosis hepática por métodos clínicos, bioquímicos y por imágenes . Revista Argentina de Endocrinología y Metabolismo, 37-46.

Marcano, R. (11 de Abril de 2015). Medicica Preventiva Santa Fe. Obtenido de http://www.medicinapreventiva.com.ve/articulos/higado_graso.html

Martínez, S. (2002). Guía de apuntes básicos para el docente de la materia técnicas de investigación. Oxaca: Grupo emergente de investigacion de Oaxaca.

Mayo Clinic. (s.f.). Enfermedad hepática del hígado graso no alcohólico. Recuperado el 11 de Agosto de 2017, de Mayo Clinic : https://www.mayoclinic.org/es-es/diseasesconditions/nonalcoholic-fatty-liver-disease/symptoms-causes/syc-20354567

My Liver Exam. . (2015). ESTEATOSIS. Obtenido de My Liver Exam: https://www.myliverexam.com/es/entender-el-higado/estado/esteatosis/

Padrón, N. (2017). Esteatosis hepática: etiología, patrones y cuantificación. Sociedad Española de Radiología Médica, 501-510.

Pinheiro, P. (s.f.). HÍGADO GRASO - ESTEATOSIS HEPÁTICA. Recuperado el 02 de Julio de 2018, de MD Saude : https://www.mdsaude.com/es/2015/10/higado-graso.html

Sampieri, R. (1998). Metodologia de la Investigacion. Mexico DF: Interamericana editores.

Stoopen-Rometti, M., Encinas-Escobar, E., Ramirez-Carmona, C., Wolpert-Barrazac, E., Kimura-Hayama, E., Sosa-Lozano, L., \& Loaeza-del Castillo, A. (2017). Diagnóstico y 
Esteatosis y Cirrosis Hepática: Métodos diagnósticos mediante Imagenología

Vol. 3, núm. 1., (2019)

Jenny Jazmín Ortega Flores; Andrea Carolina Villao Recalde; Ximena Angélica Vargas Arizaga;

Johanna Denys Suarez Orrala

cuantificación de fibrosis, esteatosis y hepatosiderosis por medio de resonancia magnética multiparamétrica. Revista de Gastroenterología de México, 32-45.

Vallejo, A., Peñafiel, M., \& Acuña, M. (2017). Utilidad de la ecografía en la detección de esteatosis hepática. Dominio de las ciencias , 684-701.

Zanin, T. (Julio de 2018). Síntomas de hígado graso y cómo eliminarlo . Obtenido de Tua Saúde.: https://www.tuasaude.com/es/grasa-en-el-higado/ 